\title{
rastram
}

\section{Cardiovascular diseases after the Great East Japan Earthquake}

The Great East Japan Earthquake, which measured 9.0 on the Richter scale, struck the Miyagi Prefecture of Japan on 11 March 2011 causing widespread death and destruction. Investigators from Sendai, Japan now show that the disaster also had prolonged cardiovascular consequences for the survivors.

Ambulance records from 4 weeks before until 16 weeks after the earthquake (11 February-30 June) were compared with those from the same periods in 2008, 2009, and $2010(n=124,152)$. Cardiovascular diseases spiked after the event, with varying timescales. Incidence of cardiopulmonary arrest rose rapidly on the day of the earthquake and then declined, whereas acute coronary syndrome events peaked 1-2 weeks after the event. Conversely, the occurrence of heart failure and pneumonia continued to be elevated $>6$ weeks after the earthquake. Acute events (stroke and cardiopulmonary arrest) spiked again after the largest aftershock on 7 April 2011.

The investigators speculate that the increased incidence of cardiovascular diseases might have been caused by "the activated sympathetic nervous system by physical and mental stresses, insufficient medications, increased salt intake from preserved foods, and elevated blood pressure and viscosity". Ute Wilbert-Lampen and Gerhard Steinbeck from Munich, Germany point out that "whatever the mechanism ... a catastrophe such as this is a challenge to any healthcare system forced to deal with increased cardiovascular disease not only for days, but for weeks and months".

Gregory B. Lim

Original article Aoki, T. et al. The Great East Japan Earthquake disaster and cardiovascular diseases. Eur. Heart J. doi:10.1093/eurheartj/ehs288 